\title{
Surface reconstructions of In-enriched InGaAs alloys
}

\author{
J. Mirecki Millunchick, A. Riposan, and B. J. Dall \\ Department of Materials Science and Engineering, University of Michigan, Ann Arbor, Michigan 48109 \\ Chris Pearson \\ Department of Computer Science, Engineering Science and Physics, University of Michigan-Flint, Flint, \\ Michigan 48502 \\ B. G. Orr \\ The Harrison M. Randall Laboratory, University of Michigan, Ann Arbor, Michigan 48109
}

(Received 3 April 2003; accepted 19 June 2003)

\begin{abstract}
The atomic structure of $\mathrm{In}_{0.81} \mathrm{Ga}_{0.19} \mathrm{As} / \mathrm{InP}$ alloy layers was examined using in situ scanning tunneling microscopy. The $(2 \times 3)$ reconstruction observed during growth by reflection high-energy electron diffraction represents a combination of surface structures, including a $\beta 2(2 \times 4)$ commonly observed on $\operatorname{GaAs}(001)$ and $\operatorname{InAs}(001)$ surfaces, and a disordered $(4 \times 3)$ that is unique to alloy systems. The proposed $(4 \times 3)$ structure is comprised of both anion and cation dimers. Empty and filled states images show that the features reverse contrast with sample bias, in agreement with the model. (C) 2003 American Institute of Physics. [DOI: 10.1063/1.1602557]
\end{abstract}

The atomic structure of compound semiconductor alloy surfaces is important for heteroepitaxial growth, as it has an impact on the subsequent microstructure of the film. Recent cross-sectional scanning tunneling microscopy (STM) data in a variety of material systems show that local variations in composition, likely initiated at the growth front, develop into lateral composition modulation. ${ }^{1-3}$ The roughness of interfaces in device structures, furthermore, has been shown to be related to the stoichiometry of the surface reconstruction. ${ }^{4}$ Finally, surface reconstructions have also been directly linked to ordering in these alloys. ${ }^{5}$

Detailed models of the atomic structure of compound semiconductor surfaces are most common for materials such as $\mathrm{GaAs},{ }^{6} \mathrm{InAs},{ }^{7} \mathrm{InP},{ }^{8}$ and $\mathrm{GaSb},{ }^{9}$ but characterization of ternary alloy surfaces has received less attention. Limited STM data are available for lattice-matched ${ }^{10}$ and strained InGaAs films. ${ }^{11,12}$ The detailed atomic structure was examined for very dilute InGaAs/GaAs films. ${ }^{13}$ InAs wetting layers on GaAs have also been examined using STM $^{14,15}$ and glancing incidence x-ray diffraction. ${ }^{16}$ Studies of In surface segregation suggest that the topmost layer is a nearly pure InAs,${ }^{17}$ however, this surface has never been imaged directly.

In this letter, we present observations of pseudomorphic $\mathrm{In}_{0.81} \mathrm{Ga}_{0.19} \mathrm{As} / \mathrm{InP}$ alloy surfaces using atomic resolution STM. We show that the surface consists of a mixture of surface reconstructions, including the $\beta 2(2 \times 4)$ common to binary compound surfaces, and a $(n \times 3)$ reconstruction, where $n=3$ or 4 , that is unique to this alloy system. The $(4 \times 3)$ reconstruction is proposed to be a mixed reconstruction; that is, it is terminated with both As and metal dimers.

The films were grown using molecular-beam epitaxy equipped with solid sources for $\mathrm{Ga}$ and $\mathrm{In}$, and a valved cracking cell for $\mathrm{As}_{4}$. $N$-type $\operatorname{InP}(001)$ substrates were heated to remove the oxide layer under an $\mathrm{As}_{4}$ overpressure beam equivalent pressure (BEP) of $12 \times 10^{-6}$ Torr until the reconstruction changed from $(2 \times 4)$ to $(4 \times 2)$, at nominally $T=535^{\circ} \mathrm{C}$, and then immediately cooled to $T=470{ }^{\circ} \mathrm{C}$. A 0.5 - $\mu$ m-thick lattice matched $\operatorname{In}_{0.53} \mathrm{Ga}_{0.47}$ As buffer layer was deposited, and the surface was annealed under these conditions for 25 min while the In cell was ramped to the temperature required to obtain the desired film composition. The 25-monolayer (ML)-thick undoped $\mathrm{In}_{0.81} \mathrm{Ga}_{0.19}$ As film was deposited at $T=460^{\circ} \mathrm{C}, \mathrm{BEP}=12 \times 10^{-6}$ Torr, and $R=1.1$ $\mathrm{ML} / \mathrm{s}$. This composition corresponds to a compressive lattice mismatch of $f=1.9 \%$. Reflection high-energy electron diffraction (RHEED) oscillations were observed over the entire deposited thickness, indicating layer-by-layer growth. At the end of growth, the films were annealed for $25 \mathrm{~min}$ at the growth conditions, and quenched under an $\mathrm{As}_{4}$ overpressure. The samples were transferred within vacuum, to a UHV STM microscope (RHK technology). The imaging conditions were typically $-3.1 \mathrm{~V}$ and $100 \mathrm{pA}$, except where noted. Performing cross correlations of each region on many images and averaging the results determined the coverage of the different reconstructions.

Figure 1 shows an STM image of a 25-ML-thick $\mathrm{In}_{0.81} \mathrm{Ga}_{0.19} \mathrm{As} / \mathrm{InP}$ film grown at $T=460{ }^{\circ} \mathrm{C}$ and annealed for $25 \mathrm{~min}$. The RHEED pattern (not shown) at the end of growth of this alloy layer was very strongly $(2 \times 3)$ in agreement with published reports. ${ }^{18}$ The surface structure as observed by STM, however, is comprised of domains of different reconstructions (Fig. 1). A large portion of the surface $(36 \pm 3 \%)$ consists of long reconstructed rows that are very similar to the $\beta 2(2 \times 4)$ reconstruction. ${ }^{7}$ Indeed, the dimensions of the unit cell are $2 a$ along the [110] and $4 a$ along the [1힐, where $a \approx 4 \AA$, the bulk lattice spacing along the $\{110\}$ directions. The $\beta 2(2 \times 4)$ reconstruction consists of a pair of dimers along the [1 $\overline{1} 0]$ separated by an As-dimer terminated trench. ${ }^{7}$ Occasionally, the second As dimer is missing, resulting in an $\alpha 2(2 \times 4)$ reconstruction, as indicated in the image $(\alpha)$. Other defects along these rows are also sporadically observed, such as kinks along the reconstruction rows $(\mathrm{K})$ or trench filling defects $(\mathrm{T})$.

The $\beta 2(2 \times 4)$ reconstruction rests upon a more disorganized surface reconstruction that covers $51 \pm 2 \%$ of the surface, and appears to be $1.5 \AA$ lower than the $\beta 2(2 \times 4)$ do- 


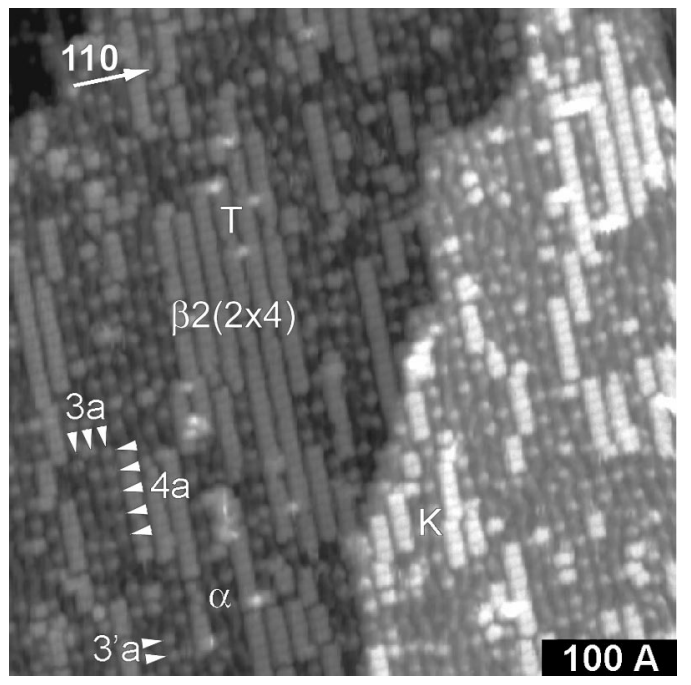

FIG. 1. STM image $(-3.1 \mathrm{~V}$ and $100 \mathrm{pA})$ of a 25-ML-thick $\mathrm{In}_{0.81} \mathrm{Ga}_{0.19} \mathrm{As} / \mathrm{InP}$ grown at $T=460{ }^{\circ} \mathrm{C}$ and annealed for $25 \mathrm{~min}$. The image shows domains of $\beta 2(2 \times 4)$ and $n \times 3$ reconstructions, and occasional regions of $\alpha 2(2 \times 4)(\alpha)$, kinks $(\mathrm{K})$ and trench-filling defects $(\mathrm{T})$. The dimer spacing is indicated for different reconstruction domains ( $\left.3 \mathrm{a}, 3^{\prime} \mathrm{a}, 4 \mathrm{a}\right)$.

mains. This reconstruction is imaged as equiaxial spots that are uniformly spaced $3 a$ along the [110] (3a in Fig. 1). There are regions where the spots are spaced $4 a$ apart along the [110] (4a), resulting in an overall reconstruction with a $(4 \times 3)$ symmetry. This $(4 \times 3)$ reconstruction has also been observed by RHEED for lattice-matched $\mathrm{In}_{0.53} \mathrm{Ga}_{0.47} \mathrm{As} / \mathrm{InP} .{ }^{10} \mathrm{~A}$ periodicity of $3 a$ is also occasionally observed ( $3^{\prime}$ a) along [ $\left.1 \overline{1} 0\right]$, thus small regions of a $(3 \times 3)$ symmetry are also present. Careful observation of the $(4 \times 3)$ and $(3 \times 3)$ domains shows that the bright spots rest upon rows aligned along the $[1 \overline{1} 0]$ separated by trenches.

This mixture of surface reconstructions is consistent with the observed $(2 \times 3)$ RHEED pattern. Even though the majority of the surface is covered by the $(4 \times 3)$ and $(3 \times 3)$ reconstructions, they are not very well ordered over long length scales along the [110], especially when compared to the $\beta 2(2 \times 4)$ reconstruction. On the other hand, the periodicity of the $4 \times 3$ reconstruction is regular along the [1 $\overline{1} 0]$ and covers $50 \%$ of the surface. This blending of the RHEED pattern as a result of multiple surface reconstructions has also been observed in InAs wetting layers on GaAs. ${ }^{19}$

Combinations of $(2 \times 4)$ and $(n \times 3)$ were observed by STM for a wide range of annealing times and In compositions. ${ }^{20}$ The $(4 \times 3)$ reconstruction observed here is similar to the so called $(1 \times 3)$ observed in InAs wetting layers on $\mathrm{GaAs},{ }^{14}$ which is a distinctly alloy reconstruction. Figure 2 shows a proposed model of the $(4 \times 3)$ reconstruction. The major unit of repetition is an As dimer pair that has an additional As dimer on top of it. This leads to the $\times 3$ periodicity along the [110]. The $4 \times$ reconstruction is obtained when a cation dimer replaces the excess As dimer. It should be noted that there is no distinction between the Group-III species in this model. However, InGaAs is known to order under certain growth conditions, ${ }^{21}$ therefore, some specificity is likely. Neither dimer is observed clearly in the STM image, most likely due to the chemical differences between the cation and anion density of states. However, the diffuse intensity between the spots along the $[1 \overline{1} 0]$ suggests (a)
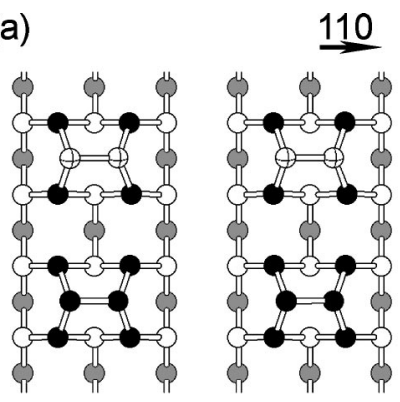

(b)

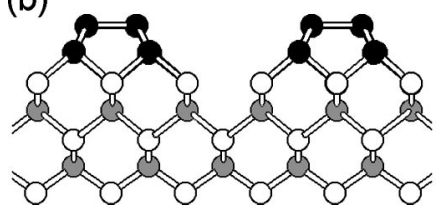

FIG. 2. Schematic of the proposed $4 \times 3$ reconstruction in (a) plan-view and (b) cross section. The gray atoms are subsurface As, the white are subsurface metal, the black are surface As, and the white crosshatched are surface metal atoms. Some subsurface atoms are omitted for clarity.

the presence of additional features on the surface. The proposed model does not obey the electron counting model, having two excess electrons per unit cell. The excess charge may be taken up by the numerous defects in the surface reconstruction ${ }^{13}$ observed in these $(n \times 3)$ regions. On the other hand, the $1.9 \%$ lattice-mismatch strain may act to stabilize this structure. ${ }^{22}$

To fully reveal the surface structure with STM, both empty and filled states images must be examined (Fig. 3). This is because when imaging the surface at just one bias polarity, both dimers are not equally resolved in the STM image due to the chemical differences between the cation and anion dimers. When imaging the filled states [Fig. 3(a)], a series of cation dimers are resolved that are spaced $16 \AA$ apart, giving rise to the four-fold periodicity along the [1 $\overline{1} 0]$. The second dimer is not observed under these imaging conditions. When the bias voltage is inverted to image the empty states [Fig. 3(b)], the contrast between the features changes, as seen in the linescans across the image [Fig. 3(c)]. In particular, the minima in the filled states image become maxima in the empty states image. This change in relative intensity of the features, which occurs for approximately $60 \%$ of the features, is consistent with the model and the typical charge states of the As and cation dimers. Occasionally, a maximum in the filled state image does not reverse in the empty state image, as seen in Fig. 3(c). This is due to the fact that the precise chemistry of the dimer, be it In-In, $\mathrm{Ga}-\mathrm{Ga}$, In-Ga, or even III-As, will have distinctly different behavior as a function of sample bias. In fact, the presence of these different types of dimers on the $(n \times 3)$ reconstruction may account for the significant disorder that is observed in the arrangement of the equiaxial spots. Ab initio calculations are necessary in order to determine the veracity of the proposed model, and the detailed positions of the In, Ga, and As species.

The resolution of the $\beta 2(2 \times 4)$ regions changes with bias, consistent with other reports, ${ }^{7}$ but the apparent height of the $\beta 2(2 \times 4)$ reconstructed regions does not. This suggests that the apparent height of the $\beta 2(2 \times 4)$ domains is indeed half a monolayer above the $(4 \times 3)$ reconstructions, and is not 

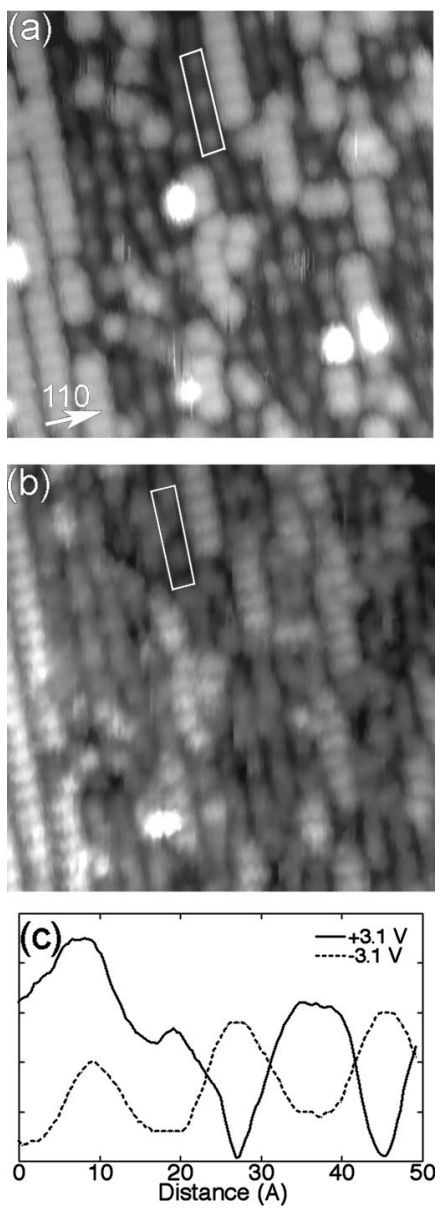

FIG. 3. STM images taken at (a) $-3.1 \mathrm{~V}$ (filled states) and $100 \mathrm{pA}$ and (b) $+3.1 \mathrm{~V}$ and $100 \mathrm{pA}$ (empty states) on the same region. (c) Line scans of the boxed regions in a and $\mathrm{b}$.

simply due to chemical differences between the various regions. Therefore, it appears that the $\beta 2(2 \times 4)$ domains nucleate on top of the $(4 \times 3)$ surface. Given this fact, it is conceivable that the $\beta 2(2 \times 4)$ reconstruction is highly enriched in In, since the larger In atoms would be able to lower their strain energy by moving out of the flat terrace. ${ }^{23}$ These domains are likely the local compositional variations that initiate lateral composition modulation. Furthermore, they may be the mechanism by which In segregates to the surface in these alloys. Indeed, preliminary data shows that the coverage of the $(2 \times 4)$ is correlated to the In composition of the film. ${ }^{20}$

In summary, $\mathrm{In}_{0.81} \mathrm{Ga}_{0.19} \mathrm{As} / \mathrm{InP}$ surfaces exhibit a surface atomic structure that consists of a mixture of reconstructions. Approximately one third of the surface is covered with $\beta 2(2$ $\times 4)$, but half the surface is covered with a disordered $(4 \times 3)$ and $(3 \times 3)$ reconstructions. The proposed $(4 \times 3)$ reconstruction is unusual in that it has both As and metal dimers on the surface. Empty and filled states images show that the apparent height of the features changes with bias, consistent with the model. This mixed termination may be the mechanism by which In segregates to the surface.

The authors gratefully acknowledge funding support from NSF grant DMR 9973352.

${ }^{1}$ B. Shin, A. Lin, K. Lappo, R. S. Goldman, M. C. Hanna, S. Francoeur, A. G. Norman, and A. Mascarenhas, Appl. Phys. Lett. 80, 3292 (2002).

${ }^{2}$ S. L. Zuo, E. T. Yu, A. A. Allerman, and R. M. Biefeld, J. Vac. Sci. Technol. B 17, 1781 (1999).

${ }^{3}$ E. T. Yu, S. L. Zuo, W. G. Bi, C. W. Tu, A. A. Allerman, and R. M. Biefeld, J. Vac. Sci. Technol. A 17, 2246 (1999).

${ }^{4}$ B. Z. Nosho, W. H. Weinberg, W. Barvosa-Carter, B. R. Bennett, B. V. Shanabrook, and L. J. Whitman, Appl. Phys. Lett. 74, 1704 (1999).

${ }_{6}^{5}$ S. Froyen and A. Zunger, Phys. Rev. B 53, 4570 (1996).

${ }^{6}$ V. Bresslerhill, M. Wassermeier, K. Pond, R. Maboudian, G. A. D. Briggs, P. M. Petroff, and W. H. Weinberg, J. Vac. Sci. Technol. B 10, 1881 (1992).

${ }^{7}$ W. Barvosa-Carter, R. S. Ross, C. Ratsch, F. Grosse, J. H. G. Owen, and J. J. Zinck, Surf. Sci. 499, L129 (2002).

${ }^{8}$ V. P. LaBella, Z. Ding, D. W. Bullock, C. Emery, and P. M. Thibado, J. Vac. Sci. Technol. A 18, 1492 (2000).

${ }^{9}$ A. S. Bracker, M. J. Yang, B. R. Bennett, J. C. Culbertson, and W. J. Moore, J. Cryst. Growth 220, 384 (2000).

${ }^{10}$ S. Ohkouchi and A. Gomyo, Appl. Surf. Sci. 132, 447 (1998).

${ }^{11}$ M. Gendry, G. Grenet, Y. Robach, P. Krapf, L. Porte, and G. Hollinger, Phys. Rev. B 56, 9271 (1997).

${ }^{12}$ L. Porte, P. Krapf, Y. Robach, M. Phaner, M. Gendry, and G. Hollinger, Surf. Sci. 352, 60 (1996).

${ }^{13}$ L. Li, B. K. Han, R. F. Hicks, H. Yoon, and M. S. Goorsky, Ultramicroscopy 73, 229 (1998).

${ }^{14}$ J. G. Belk, C. F. McConville, J. L. Sudijono, T. S. Jones, and B. A. Joyce, Surf. Sci. 387, 213 (1997).

${ }^{15}$ V. Bresslerhill, A. Lorke, S. Varma, P. M. Petroff, K. Pond, and W. H. Weinberg, Phys. Rev. B 50, 8479 (1994).

${ }^{16}$ M. Sauvagesimkin, Y. Garreau, R. Pinchaux, M. B. Veron, J. P. Landesman, and J. Nagle, Phys. Rev. Lett. 75, 3485 (1995).

${ }^{17}$ J. M. Moison, C. Guille, F. Houzay, F. Barthe, and M. Vanrompay, Phys. Rev. B 40, 6149 (1989).

${ }^{18}$ J. M. Moison, C. Guille, and M. Bensoussan, Phys. Rev. Lett. 58, 2555 (1987).

${ }^{19}$ C. F. Mcconville, T. S. Jones, F. M. Leibsle, S. M. Driver, T. C. Q. Noakes, M. O. Schweitzer, and N. V. Richardson, Phys. Rev. B 50, 14965 (1994).

${ }^{20}$ J. M. Millunchick, A. Riposan, B. J. Dall, C. A. Pearson, and B. G. Orr (unpublished).

${ }^{21}$ A. Zunger and S. Mahajan, Atomic Ordering and Phase Separation in Epitaxial III-V Alloys, in Handbook on Semiconductors, edited by S. Mahajan (Elsevier Science, New York, 1994), p. 1399.

${ }^{22}$ A. Ohtake, J. Nakamura, S. Tsukamoto, N. Koguchi, and A. Natori, Phys. Rev. Lett. 89, 206102 (2002).

${ }^{23}$ J. E. Guyer and P. W. Voorhees, J. Cryst. Growth 187, 150 (1998). 